\title{
BMJ Open How does overall hospital satisfaction relate to patient experience with nursing care? a cross-sectional study in China
}

\author{
Xiao Chen, ${ }^{1}$ Yuxia Zhang (1) , ${ }^{1}$ Wei Qin, ${ }^{1}$ Zhenghong Yu, ${ }^{2}$ JingXian Yu, ${ }^{3}$ Ying Lin, ${ }^{4}$ \\ XiaoRong $\mathrm{Li}^{5}{ }^{5}$ Zheng Zheng, ${ }^{6}$ Ying Wang ${ }^{5}$
}

To cite: Chen $X$, Zhang $Y$, Qin W, et al. How does overall hospital satisfaction relate to patient experience with nursing care? a cross-sectional study in China. BMJ Open 2022;12:e053899. doi:10.1136/ bmjopen-2021-053899

- Prepublication history and additional supplemental material for this paper are available online. To view these files, please visit the journal online (http://dx.doi.org/10.1136/ bmjopen-2021-053899).

Received 02 June 2021 Accepted 30 November 2021

Check for updates

(C) Author(s) (or their employer(s)) 2022. Re-use permitted under CC BY-NC. No commercial re-use. See rights and permissions. Published by BMJ.

${ }^{1}$ Department of Nursing, Zhongshan Hospital Fudan University, Shanghai, China ${ }^{2}$ Department of Surgery, Zhongshan Hospital Fudan University, Shanghai, China ${ }^{3}$ Department of Liver Disease, Zhongshan Hospital Fudan University, Shanghai, China ${ }^{4}$ Department of Cardiology, Zhongshan Hospital Fudan University, Shanghai, China ${ }^{5}$ Department of Internal Medicine, Zhongshan Hospital Fudan University, Shanghai, China

${ }^{6}$ Department of Respiratory, Zhongshan Hospital Fudan University, Shanghai, China

Correspondence to

Dr Yuxia Zhang;

zhang.yuxia@zs-hospital.sh.cn

\section{ABSTRACT}

Objective To determine how patient experience with nursing care influence patient satisfaction with overall hospital services.

Design This was a cross-sectional study.

Setting Inpatients were consecutively recruited at the national hospital (with 2000 beds) in Shanghai, China. Participants The inclusion criteria were as follows: (1) hospitalised for 2 days or more; (2) able to read and understand Chinese; and (3) aged 18 years old or above. Patients with mental health problems were excluded. 756 patient surveys distributed among 36 wards were analysed. The mean age of participants in the study was $57.7(\mathrm{SD}=14.5)$ and ranged from 18 to 80 years. Most participants were men (61.5\%) and ever married (94.6\%). Primary and secondary outcome measures Patient experience with nursing care, meaning the sum of all interactions between patients and nurses, was measured using the self-designed questionnaire, which was developed by patient interviews, literature analysis and expert consultation. The overall patient satisfaction question was measured with a 10-point response option ranging from 1 to 10 .

Results A linear relationship between the patient experience with nursing care and overall patient satisfaction was observed. The patient experience with nursing care was significantly associated with overall satisfaction in the crude model and in the adjusted models. Even after adjusting for six sociodemographic and three disease-related factors, the patient experience with nursing care explained $34.9 \%$ of the variation in overall patient satisfaction.

Conclusions This study showed that patient experience with nursing care was an important predictor for overall patient satisfaction.

\section{INTRODUCTION}

In the age of patient-centred care, as valuebased care expands, patient satisfaction has become a key indicator in assessing healthcare quality and hospital performance ${ }^{1}$ and is being used more frequently to determine hospital performance and hospital reimbursement. ${ }^{23}$ Patients who are satisfied with the healthcare system are more willing to comply with medical orders and treatments, ${ }^{4}$ are more likely to return to the healthcare

\section{Strengths and limitations of this study}

This study used a valid and specific questionnaire of patient experience with nursing care made by patient interviews, literature analysis and expert consultation to investigate patient experience with nursing care.

- This study quantitatively analysed the impact of patient experience with nursing care on overall patient satisfaction.

- This study first surveyed patient experience with nursing care systematically and comprehensively in China.

- This was a single-centre study and our findings therefore may not be generalised.

- This study did not survey hospital-unit-related characteristics, such as the organisation's patientcentred culture and nurses' practice environment. These variables were not available in our data sample but might be associated with patient experience with nursing care and also have an effect on overall patient satisfaction.

organisation for future care and are more likely to recommend healthcare services to their family members and friends. ${ }^{5}$ As the healthcare quality improvement action plan proliferated internationally, the National Health Commission of the People's Republic of China posted an announcement implementing the National Healthcare Improvement Initiative in January 2015, with the overall goal of improving the patient satisfaction on a national level. ${ }^{6}$

Recognising factors that influence overall patient satisfaction will help improve medical care. A large body of research has identified the factors that account for the variations in patient satisfaction. ${ }^{7}$ However, such studies have largely focused on patient characteristics, such as age,${ }^{8}$ gender, ${ }^{9}$ race/ethnicity, ${ }^{10}$ financial status ${ }^{11}$ and organisational factors ${ }^{12}{ }^{13}$; additionally, these studies have inconsistent findings and explained only a small fraction of the variance in patient satisfaction. 
In recent years, patient experience has been increasingly used to evaluate the quality of healthcare. ${ }^{14}$ Patient experience is defined as 'the sum of all interactions, shaped by an organisation's culture, that influence patient perceptions across the continuum of care' ${ }^{15}$ Patient experience measures the structures and processes of care, while patient satisfaction survey serves as a patient-reported outcome measure. ${ }^{15}$ The causal link between structure, process and outcome might be expected theoretically. However, patient satisfaction is subjective and obscure, and dependent on patients' expectations, fulfilment of expectations, actual experiences, health outcome and other individual factors. Therefore, several studies have explored the relationship between patient experience and patient satisfaction with the healthcare system, ${ }^{16-18}$ with an attempt to determine to what extent that patient experience affects patient satisfaction, considered that patient experience can provide tangible feedbacks to current care delivery and these feedbacks are amendable and actionable by providers to improve quality of healthcare, whereas other factors such as patients' expectations and individual characteristics are hard to change.

Nurses are a vital and central part of the healthcare system, ${ }^{19}$ accounting for nearly half of the global health workforce and spending more time with patients than any other medical professionals. ${ }^{20}$ According to the data from the latest China Health Statistics Yearbook issued by the National Health Commission of the People's Republic of China, as of the end of 2020, the number of nurses in China reached 4.7 million, accounting for $44.1 \%$ of the total number of healthcare professionals. ${ }^{21}$ In theory, patient experience with nursing care, as a process indicator, reflects interactions between patients and nurses and has an important impact on overall satisfaction with hospital care. ${ }^{22}{ }^{23}$ In the study of Bjertnaes, ${ }^{18} 13$ variables were significantly associated with overall patient satisfaction with hospitals, and the results of the regression model showed that the most important predictor of patient satisfaction with hospitals was patient experiences with nursing care. Similarly, Schmidt found that the perception of nursing care received was the only significant predictor of overall satisfaction with the hospital experience. $^{24}$

However, in terms of using these patient experience data to improve nursing care, existing studies have not offered enough feedback due to the low representation of nursing care in these patient experience surveys. ${ }^{1718}$ Most patient experience scales include a limited number of items related to nursing and fail to provide thorough and detailed insight into nursing care from patients' perspectives. For instance, the study of Bjertnaes ${ }^{18}$ included only four items related to nursing care, and the study of Min $e t a l^{12}$ included only two items related to nursing care. Therefore, to what extent patient experience with nursing care explains satisfaction with the healthcare system remains unclear.

We hypothesise that patient experience with nursing care accounts for a considerable portion of the unexplained variation in health system satisfaction after adjustments for the demographic profile, health and organisational factors with which patient satisfaction is usually associated. Understanding the association between patient experience with nursing care and patient satisfaction may help in using the results to improve nursing services, resulting in better patient satisfaction. Therefore, the purpose of this study was to determine how patient experience with nursing care influence satisfaction among patients.

\section{METHODS \\ Design}

This study is a cross-sectional survey and is reported according to the "The Strengthening the Reporting of Observational Studies in Epidemiology (STROBE) Statement for reporting observational studies' obtained from the EQUATOR Network website. ${ }^{25}$

\section{Setting}

Inpatients were consecutively recruited from July 2020 to August 2020 in Zhongshan Hospital of Fudan University, which is the largest academic hospital (with 2000 beds) in Shanghai, China.

\section{Sample and participants}

We calculated the sample size according to the requirements for multivariate analysis, which demands the sample size be $5-10$ times the number of variables. ${ }^{26}$ There were 33 items in the questionnaire and 22 patients and organisational characteristics. Therefore, the sample size was required to be 660 with an estimated $20 \%$ non-response rate. During the study period, a total of 767 inpatients were eligible to participate in the study, 7 patients refused to participate $(0.9 \%)$, and 4 patients' questionnaires were incomplete $(0.5 \%)$. Finally, 756 patients $(98.6 \%)$ were analysed. The inclusion criteria were as follows: (1) hospitalised for 2 days or more; (2) able to read and understand Chinese; and (3) aged 18 years old or above. Patients with mental health problems, such as dementia, schizophrenia and severe depression, were excluded. Eligible patients were invited to participate in the study. When a patient showed an interest in participating, a recruitment letter explaining the aim, process and ethical considerations of this study was sent to them. To gain a broad and representative understanding of the patient experience, we varied the recruitment sites. A total of 36 wards were included, including 16 internal medical wards and 20 surgical wards, followed by cardiology, hepatology, respiratory department, gastroenterology, haematology, oncology, nephrology, orthopaedics, urology, neurology, obstetrics and gynaecology, endocrinology, otorhinolaryngology and hepatobiliary surgery department, from each of which accounted for at least $3 \%$ of the patients in our sample. 


\section{Measures}

Patient characteristics

The following characteristics were collected: age, gender, ethics, religion, educational level, household monthly income per capita, family residence, medical assurance, primary caregiver, primary disease diagnosis, number of admissions within 1 year and length of hospital stay. The section for disease diagnosis consisted of 10 categories: (1) cardiovascular diseases, (2) pulmonary diseases, (3) diseases of the digestive system, (4) diseases of the musculoskeletal system, (5) endocrine/metabolic diseases, (6) neurological diseases, (7) diseases of ophthalmology, (8) diseases of the urinary system, (9) diseases of the haematological system and (10) other diseases, including allergies.

\section{Patient experience with nursing care}

Patient experience with nursing care was measured by the inpatient experience of nursing care questionnaire, which was self-designed to evaluate patients' perceptions of quality of nursing care in Chinese hospitals. After a scoping review of current research results concerning patient experience with nursing care, 15 semi-structured in-depth interviews with 8 men and 7 women were conducted to obtain insights into patientperceived important elements of nursing care. Example questions are 'What aspects of nursing care do you feel are important?' and 'What do you see as the nurses' role when you receive health services?'. The draft items of the questionnaire were generated by interviews and literature analysis. Then, to select the most suitable items to be retained in the questionnaire, the content validity of the items was evaluated by 15 experts in the fields of patient management and quality of care, and items were deleted if the content validity index was less than 0.8 . Finally, we conducted a pilot survey and found the Cronbach's $\alpha$ of the questionnaire was 0.84 , and the split-half reliability was 0.75 .

The final questionnaire consisted of 33 items assessing eight dimensions of patients' perception of nursing care (online supplemental material 1): (1) Coordination of care (three items), for example, the process of admission. (2) Physical environment (three items), for example, the cleanliness of the ward. (3) Information and education (seven items), for example, the information about how to conduct scientific lifestyles. (4) Emotional support (four items), for example, nurses' response to patients' anxiety and fear. (5) Technical competencies (two items), for example, proficiency in performing nursing procedures. (6) Monitoring the progress of diseases (four items), for example, monitoring the vital signs. (7) Responding requests (three items), for example, the waiting time after pressing the call button. (8) Patient safety and privacy protection (seven items), for example, treating patients' information confidentially. Most of the items were assessed by a 5-point Likert scale ranging from 'never' to 'always', where 'never' $=1$, 'occasionally' $=2$, 'sometimes' $=3$, 'usually' $=4$, and 'always' $=5$. Response options ranged from 'strongly disagree' and 'strongly agree' for the admission process and discharge plan. For each item, the patients were offered the option of indicating whether it was not relevant. Each dimension score was determined by adding the scores of all items that corresponded to that dimension and dividing it by the number of items. The total inpatient experience score was the mean of all eight dimension scores.

\section{Patient satisfaction}

The overall patient satisfaction question was "All in all, were you satisfied with the care and treatment you received at the hospital?', with a 10-point response option ranging from 1 to 10 (with 1 labelled 'not at all satisfied' and 10 labelled 'to a very large extent satisfied').

\section{Data collection}

Eligible patients were invited to participate in the study. After informed consent was given, all data were obtained by trained investigators. Characteristics included in the hospital information system, such as gender, age and diagnosis, were collected by checking the information system, while characteristics related to family income, literacy level and number of hospital admissions were assessed by interviewing patients and their family members. The timing of collecting the patients' feedback may affect their response to the questionnaires because some of them may worry that negative appraisals about their hospital experience and satisfaction would affect the treatment and care they received during hospitalisation, and thus they might be unwilling to provide negative feedback. To encourage the participants to respond frankly, the patient experience with nursing care survey and the overall patient satisfaction survey were taken on the patients' discharge day, and the nursing staff did not administer the survey.

\section{Data analysis}

Statistical analyses were conducted using IBM-SPSS software V.22 (IBM Corp), Empower (R) (www.empowerstats. com, X\&Y solutions) and R statistical software. Descriptive analysis was performed for participants' characteristics and their responses to items about satisfaction and experience. Values were expressed as the mean and SD for continuous variables or percentages for categorical variables. Multiple regression models were used to analyse the effects of patient experience with nursing care and other variables on the overall patient satisfaction. Independent variables were selected based on evidence in previous studies $^{6-8}$ showing a significant relation to overall patient satisfaction and we also included other variables based on our clinical experience. To ensure the stability of the model, and determine whether the relationship between patient experience with nursing care and patient overall satisfaction would be weakened after adjusting different variables, we chose different kinds of variables into the model successively. Model 1 was adjusted for age, sex, residence, literacy level, household monthly income per 
capita, type of medical assurance; model 2 was adjusted for age, sex, residence, literacy level, household monthly income per capita, type of medical assurance, diagnosis, number of admissions within 1 year, length of hospital stay. We also conducted the subgroup analyses test whether the relationship between patient experience with nursing care and overall patient satisfaction was valid among different populations. Non-ordinal categorical variables and ordinal categorical variables with non-equidistant data were transformed into dummy variables. The probability was considered significant when $\mathrm{p}<0.05$. No missing data imputation methods were used.

\section{Patient and public involvement statement}

It was not appropriate or possible to involve patients or the public in the design, or conduct, or reporting, or dissemination plans of our research.

\section{RESULTS}

\section{Sociodemographic and clinical characteristics of the study} participants

A total of 756 participants with a mean age of 57.7 years were recruited. Of these, $61.5 \%(465 / 756)$ were men, and $39.9 \%(302 / 756)$ were diagnosed with cancer. The detailed demographic and clinical characteristics of the participants are shown in table 1.

\section{Patient experience with nursing care}

The total patient experience score was 4.54 (0.37). The scores of each item are presented in table 2. The lowest scores were related to 'information and communication' (4.34 \pm 0.52$)$, 'coordination of care' $(4.42 \pm 0.53)$ and 'emotional support' $(4.56 \pm 0.45)$. Patients had better experiences with 'patient safety and privacy protection' $(4.65 \pm 0.39)$, 'technical competencies' $(4.64 \pm 0.38)$ and 'responding requests' $(4.63 \pm 0.41)$.

\section{Patient satisfaction with hospital services}

The overall patient satisfaction item was skewed toward a positive assessment: 9.2 on a scale of $1-10$, where 10 represents the best score. Of those who responded, $52.0 \%$ were satisfied with the hospital services to a very large extent. Only $1.9 \%$ reported being satisfied to only a small extent, and $0.3 \%$ were not at all satisfied with the hospital services.

\section{Relationships between patient experience with nursing care and overall patient satisfaction}

A linear relationship between the patient experience of nursing care and overall patient satisfaction was observed after adjusting for age, sex, household monthly income per capita, literacy level, residence, medical insurance, length of hospital stay, number of admissions within 1 year and primary diagnosis (figure 1). Table 3 presents the results of multivariate regression for the effects of patient experience with nursing care on the patients' overall satisfaction with hospital services. The patient experience with nursing care was significantly associated
Table 1 Socio-demographic and clinical characteristics of study participants

\begin{tabular}{lr}
\hline Characteristics & \multicolumn{1}{l}{ Value } \\
\hline Sex, $\mathbf{n}(\%)$ & \\
\hline Male & $465,61.5$ \\
\hline Female & $291,38.5$ \\
\hline Age, mean \pm SD & $57.7 \pm 14.5$ \\
\hline Marital status, $\mathbf{n}(\%)$ & \\
\hline Single & $41,5.4$ \\
\hline Ever married & $715,94.6$ \\
\hline Literacy level, $\mathbf{n}(\%)$ & \\
\hline Primary education or below & $109,14.4$ \\
\hline Secondary education & $454,60.1$ \\
\hline College education or above & $193,25.5$ \\
\hline Household monthly income per capita, $\mathbf{n}(\%)$ \\
\hline$<5000$ RMB & $288,38.1$ \\
\hline 5000-9999 RMB & $293,38.8$ \\
\hline >10000 RMB & $175,23.1$ \\
\hline
\end{tabular}

Main source of medical expense, $\mathbf{n}(\%)$

Urban medical insurance $\quad 505,66.8$

Rural medical insurance

$163,21.6$

Commercial medical insurance

$8,1.0$

Personal funds $80,10.6$

Residence, $\mathbf{n}(\%)$

Rural areas 204, 27

Urban areas $\quad 416,55$

Rural-urban fringe areas $\quad 136,18$

Diagnosed with cancer, $\mathrm{n}(\%)$

Yes 302, 39.9

No

$454,60.1$

Number of hospital admissions within $\mathbf{1}$ year, $\mathbf{n}(\%)$

$\begin{array}{lc}1 & 457,60.4 \\ 2 & 121,16.0 \\ 3 & 58,7.7 \\ >3 & 120,15.9\end{array}$

\begin{tabular}{ll} 
Units type, $\mathbf{n}(\%)$ & \\
Cardiology & $78,10.3$ \\
Hepatology & $72,9.5$ \\
\hline Respiratory department & $65,8.6$ \\
Gastroenterology & $64,8.5$ \\
\hline Haematology & $53,7.0$ \\
Oncology & $53,7.0$ \\
Nephrology & $51,6.7$ \\
Orthopaedics & $46,6.1$ \\
Urology & $44,5.8$ \\
Neurology & $43,5.7$ \\
Obstetrics and gynaecology & $39,5.2$ \\
\hline
\end{tabular}

Continued 
Table 1 Continued

\begin{tabular}{lc}
\hline Characteristics & Value \\
\hline Thoracic surgery department & $37,4.9$ \\
Endocrinology & $35,4.6$ \\
Otorhinolaryngology & $28,3.7$ \\
Hepatobiliary surgery department & $27,3.6$ \\
Breast department & $21,2.8$ \\
\hline
\end{tabular}

with overall satisfaction in the crude model and in the adjusted models. Even after adjusting for six sociodemographic and three disease-related factors in model 2, the patient experience with nursing care was still significantly associated with overall patient satisfaction $(\beta=1.257$, adjusted $\mathrm{R}^{2}=34.9 \%, \mathrm{p}<0.001$ ).

\section{Subgroup analysis of the relationship between patient experience with nursing care and overall patient satisfaction}

The subgroup analysis is presented in figure 2. No significant heterogeneity was found among analysed subgroups stratified according to age, sex, residence, literacy level, household monthly income per capita, type of medical assurance, primary diagnosis, number of admissions within 1 year and length of hospital stay.

\section{DISCUSSION}

The aim of our study was to analyse the effects of patient experience with nursing care on overall patient satisfaction. The results showed a linear relationship between patient experience with nursing care and overall patient satisfaction after the adjustment for age, sex, family monthly income, educational level, residence, medical insurance, length of hospital stay, number of admissions, primary diagnosis (figure 1). The patient experience with nursing care explained $34.9 \%$ of the variance in overall patient satisfaction. This finding was consistent with previous studies, ${ }^{24}{ }^{27}$ which showed that the most important predictor of patient satisfaction with hospitals was patient experience with nursing care. The variance in overall patient satisfaction that patient experience with nursing care explained in our study was larger than that in study of Bjertnaes. ${ }^{18}$ The possible reason may be the different tools we used. The study of Bjertnaes ${ }^{18}$ included only four items relating to nursing and had low representation of nursing care, failing to provide thorough and detailed insight into nursing care from the patients' perspectives, while our study developed and used a questionnaire of patient experience with nursing care through patient interviews, literature analysis and expert consultation, which consisted of 33 items assessing eight dimensions of the patients' perception of nursing care and had good validity and reliability. Therefore, the survey tool used in our study had a high representation of patientperceived nursing care.

To our knowledge, this is the first study in China to survey patient experience with nursing care and to analyse its relationship with overall patient satisfaction. Recently, there has been a growing interest in using patient experience to assess and improve the performance of the healthcare system in China. ${ }^{11}$ However, nursing seems to be overlooked in this growing trend. ${ }^{28}$ Our study showed that patients had better experiences with 'patient safety and privacy protection', 'technical competencies' and 'responding requests'. The year of 2021 is the 11th anniversary of the launch of Quality Care Demonstration Project by the Chinese government, aiming at improving satisfaction of patients, society and government through high-quality nursing care. ${ }^{29}$ Driven by the implementation of the 'high-quality care project', Chinese nursing services have continued to be improved regrading patients' physical care.

However, there should be recognition of the potential need for psychological and emotional support, as well as of the importance of meeting communication and information needs. The result of our study showed that patients had worse experience with 'information and communication', 'coordination of care' and 'emotional support', which was consistent with study of Senarat and Gunawardena. ${ }^{30}$ As patients' healthcare demands increase, they are no longer satisfied with passively receiving care; instead, they are eager to become fully involved in the treatment and recovery process. ${ }^{31}$ Additionally, nurses spend the most time with them among all medical professionals. In addition to direct care providers, nurses are also expected to act as navigators coordinating all aspects of care and promoting patient-centred care. Therefore, coordination of care is a fundamental and core value of nursing care, a predictor of quality and a known predictor of patient satisfaction with healthcare. ${ }^{32}$ Humanistic care is an indispensable characteristic of nursing services. Numerous studies have demonstrated that patients' health outcomes can be improved much more significantly when caring behaviours are performed with empathy and compassion. $^{33} 34$ The study of Karam ${ }^{15}$ also showed that tactics alone, such as bedside shift reports, health education and follow-up phone calls after discharge, were insufficient, while meaningful strategies to create a positive organisational culture were vital drivers to promote a successful patient experience. However, most healthcare institutions in China are task-oriented, and the delivery of nursing care is streamlined with standardised processes, protocols and paths. These practices result in the fragmented nursing care, and patients receive less psychological care and more technical care from nurses, which negatively influences patient experience. Efforts should be made by hospital administrators and nursing managers to overcome the tendency to streamline the care delivery by standardised processes and determine how these patientperceived attributes of nursing care can be developed and rooted in the daily practice through organisational changes, culture shaping and staff education.

Compared with the other determinants that influence overall patient satisfaction with hospital services, such as the reputation and the image of hospitals, education and socioeconomic status of the patients and length of stay, ${ }^{6}$ patient 
Table 2 Percentage distribution of items for experience with nursing care in participants $(\mathrm{n}, \%)$

\begin{tabular}{|c|c|c|c|c|c|c|}
\hline Domain & Items & $\begin{array}{l}\text { Never/ } \\
\text { strongly } \\
\text { disagree }\end{array}$ & $\begin{array}{l}\text { Occasionally/ } \\
\text { disagree }\end{array}$ & $\begin{array}{l}\text { Sometimes/ } \\
\text { neutral }\end{array}$ & $\begin{array}{l}\text { Often/ } \\
\text { agree }\end{array}$ & $\begin{array}{l}\text { Always/ } \\
\text { strongly agree }\end{array}$ \\
\hline \multirow{2}{*}{$\begin{array}{l}\text { Coordination of } \\
\text { care }\end{array}$} & Nurses provided well-organised admission process & $0(0.0)$ & $5(0.7)$ & $31(4.1)$ & $317(41.9)$ & $403(53.3)$ \\
\hline & Nurses provided well-organised discharge plan & $3(0.4)$ & $7(0.9)$ & $89(11.8)$ & $336(44.4)$ & $321(42.5)$ \\
\hline $\begin{array}{l}\text { Physical } \\
\text { environment }\end{array}$ & Nurses provided an ordered ward environment & $2(0.3)$ & $6(0.8)$ & $26(3.4)$ & $246(32.5)$ & $476(63.0)$ \\
\hline \multirow[t]{5}{*}{$\begin{array}{l}\text { Information and } \\
\text { communication }\end{array}$} & $\begin{array}{l}\text { Nurses informed me about usage, dosage and side } \\
\text { effects of medicines }\end{array}$ & $0(0.0)$ & $0(0.0)$ & $14(1.9)$ & $347(45.9)$ & $395(52.2)$ \\
\hline & Nurses helped me better know the disease & $0(0.0)$ & $1(0.1)$ & $70(9.3)$ & $393(52.0)$ & $292(38.6)$ \\
\hline & Nurses informed me about results of tests & $14(1.9)$ & $110(14.5)$ & $145(19.2)$ & $382(50.5)$ & $105(13.9)$ \\
\hline & $\begin{array}{l}\text { Nurses provided health information through multiple } \\
\text { routes }\end{array}$ & $0(0.0)$ & $0(0.0)$ & $30(4.0)$ & $272(35.9)$ & $454(60.1)$ \\
\hline & $\begin{array}{l}\text { Nurses provided relevant instructions before } \\
\text { implementing medical procedures }\end{array}$ & $0(0.0)$ & $16(2.1)$ & $27(3.6)$ & $331(43.8)$ & $382(50.5)$ \\
\hline \multirow[t]{4}{*}{ Emotional support } & Nurses treated me patiently & $0(0.0)$ & $1(0.1)$ & $14(1.9)$ & $288(38.1)$ & $453(59.9)$ \\
\hline & Nurses treated me with respect & $0(0.0)$ & $2(0.3)$ & $4(0.5)$ & $239(31.6)$ & $511(67.6)$ \\
\hline & Nurses' behaviours made me feel cared for & $0(0.0)$ & $0(0.0)$ & $38(5.0)$ & $298(39.4)$ & $420(55.6)$ \\
\hline & $\begin{array}{l}\text { Nurses helped me manage the anxiety, stress, fears I } \\
\text { had about my illness }\end{array}$ & $1(0.1)$ & $4(0.5)$ & $23(3.0)$ & $307(40.6)$ & $421(55.8)$ \\
\hline $\begin{array}{l}\text { Technical } \\
\text { competencies }\end{array}$ & Nurses were proficient in venipuncture procedures & $0(0.0)$ & $1(0.1)$ & $5(0.7)$ & $261(35.8)$ & $463(63.4)$ \\
\hline \multirow[t]{3}{*}{$\begin{array}{l}\text { Responding } \\
\text { requests }\end{array}$} & $\begin{array}{l}\text { Nurses could come and see me in time after pressing } \\
\text { the call button }\end{array}$ & $4(0.5)$ & $2(0.3)$ & $20(2.6)$ & $88(11.6)$ & $642(84.9)$ \\
\hline & Nurses dealt with my requests promptly & $0(0.0)$ & $0(0.0)$ & $29(3.8)$ & $304(40.2)$ & $423(56.0)$ \\
\hline & $\begin{array}{l}\text { Nurses responded to my suggestions or complaints } \\
\text { seriously }\end{array}$ & $1(0.1)$ & $1(0.1)$ & $13(1.7)$ & $280(37.0)$ & $461(61.0)$ \\
\hline \multirow[t]{7}{*}{$\begin{array}{l}\text { Patients safety and } \\
\text { privacy protection }\end{array}$} & $\begin{array}{l}\text { Nurses could handle in time when my condition } \\
\text { experienced changes }\end{array}$ & $0(0.0)$ & $1(0.1)$ & $13(1.7)$ & $282(37.3)$ & $460(60.8)$ \\
\hline & $\begin{array}{l}\text { Nurses informed me about how to prevent the risk } \\
\text { events, such as falling and dropping from the bed }\end{array}$ & $3(0.4)$ & $4(0.5)$ & $21(2.8)$ & $251(33.2)$ & $477(63.1)$ \\
\hline & $\begin{array}{l}\text { Nurses clearly introduced the use of safety protection } \\
\text { equipment, such as the emergency call button in the } \\
\text { toilet }\end{array}$ & $5(0.7)$ & $2(0.3)$ & $26(3.4)$ & 249 (32.9) & $474(62.7)$ \\
\hline & $\begin{array}{l}\text { Nurses verified my identify when performing nursing } \\
\text { procedures }\end{array}$ & $1(0.1)$ & $0(0.0)$ & $1(0.1)$ & $231(30.6)$ & $523(69.2)$ \\
\hline & $\begin{array}{l}\text { Nurses applied hand disinfection before performing } \\
\text { nursing procedures }\end{array}$ & $0(0.0)$ & $1(0.1)$ & $55(7.3)$ & $186(24.6)$ & $514(68.0)$ \\
\hline & $\begin{array}{l}\text { Nurses provided protective measures when performing } \\
\text { nursing procedures in private body parts }\end{array}$ & $2(0.3)$ & $0(0.0)$ & $1(0.1)$ & 99 (13.1) & $654(86.5)$ \\
\hline & Nurses treated my information confidentially & $1(0.1)$ & $4(0.5)$ & $6(0.8)$ & $248(32.8)$ & $497(65.7)$ \\
\hline
\end{tabular}



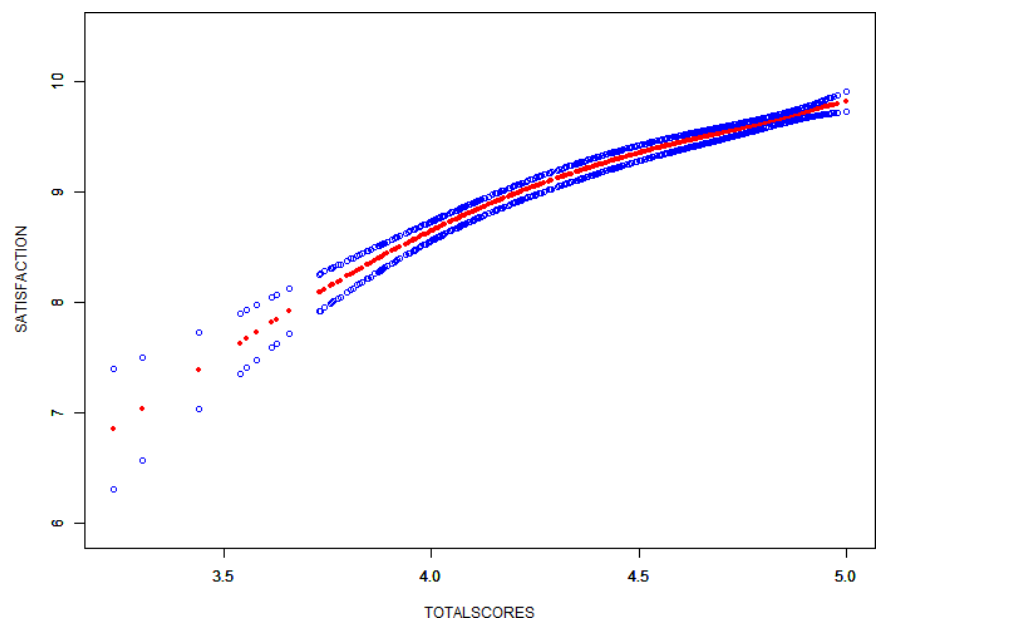

Figure 1 The relationship between patient experience with nursing care and overall patient satisfaction.

experience with nursing care is amendable and actionable. For instance, organising an afternoon ward round by nurses to address the communication needs of patients and hanging a poster to share patient feedback with the medical team have been proven to be efficient ways to facilitate good experiences with communication. ${ }^{35}$ Understanding the importance of patient experience with nursing care would enable nursing managers and nursing practitioners to have a better understanding of current problems with healthcare delivery, push for continuous improvement, redesign the delivery of services and help professionals reflect on their practice.

\section{Limitations}

This was a single-centre study and our findings therefore may not be generalised. However, our hospital is a national large general hospital and the nursing services model has a leading role around the country, therefore, for the Chinese region, our results can be regarded as representative to a considerable extent. Moreover, even though we had consider several variables which are likely related to patient outcomes, we might have omitted other hospital-unit-related characteristics, such as the organisation's patient-centred culture and nurses' practice environment. These variables were not available in our data sample but might be associated with patient experience with nursing care and also have an effect on overall patient satisfaction. Further researches are needed to analyse the external factors that could have influenced patient experience with nursing care.

\section{CONCLUSION}

This study provides the first evidence of the importance of nursing care in improving overall patient satisfaction, and demonstrates that nurses have the huge potential to contribute to the patient-centred healthcare system and nursing should be more involved in the healthcare quality improvement. Understanding the importance of patients' perception of nursing services delivery would 


\begin{tabular}{l} 
Subgroup \\
Overall \\
Age, years \\
$<60$ \\
$>=60$ \\
Sex \\
Male \\
Female \\
Literacy \\
Primary education or below \\
Secondary education \\
College education or above \\
Household monthly income per capita, RMB \\
$<5000$ \\
$5000-9999$ \\
10000 \\
Residence \\
Rural areas \\
Urban areas \\
Rural-urban fringe areas \\
Main source of medical expense \\
Urban medical insurance \\
Rural medical insurance \\
Commercial medical insurance \\
Personal funds \\
Diagnosed with cancer \\
Yes \\
No \\
Number of hospital admission within 1 year \\
$>1$ \\
Length of hospital day \\
57 days \\
$>7$ days \\
\hline .
\end{tabular}

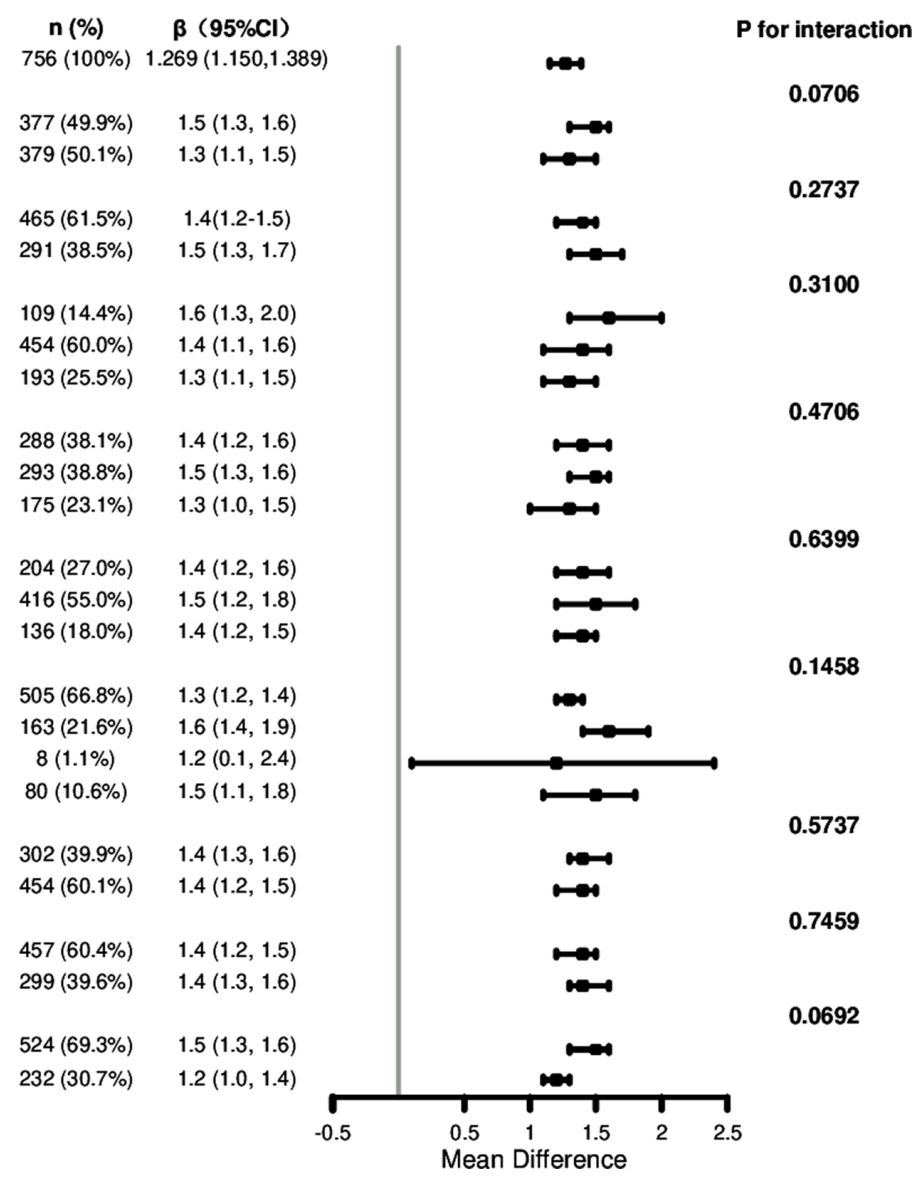

Figure 2 Subgroup analysis for effect of patient experience with nursing care on overall satisfaction with hospital services.

enable nursing managers and nursing practitioners to have better understanding of current problems with healthcare delivery, push for continuous improvement, redesign the delivery of services and help professionals reflect on practice modern.

Contributors $\mathrm{YZ}$ is responsible for the overall content as the guarantor. $\mathrm{XC}$ and $Y Z$ contributed to the study conception and design. Material preparation, data collection and analysis were performed by $X C, Y Z, W Q, Z Y, J Y, Y L, X L, Z Z$ and $Y W$. The first draft of the manuscript was written by $X C$ and $Y Z, W Q, Z Y, J Y, Y L, X L, Z Z$ and $\mathrm{YW}$ commented on and revised previous versions of the manuscript. All authors read and approved the final manuscript, and agreed to be accountable for all aspects of the work in ensuring that questions related to the accuracy or integrity of any part of the work were appropriately investigated and resolved.

Funding This work was supported by the Zhongshan Hospital of Fudan University under Award Number 20208ZSFZ02, and the funder had no role in the design of the study, the collection or analysis of data or the decision to publish.

Competing interests We had no associations with commercial entities that provided support for the work reported in the submitted manuscript. We had no associations with commercial entities that could be viewed as having an interest in the general area of the submitted manuscript. We had no similar financial associations involving their spouse or their children under 18 years of age. We had no non-financial associations that may be relevant to the submitted manuscript.

Patient consent for publication Consent obtained directly from patient(s).

Ethics approval The study was approved by the Ethics Committee of Zhongshan Hospital of Fudan university (Approval number B2020-074) and was performed in accordance with the ethical standards of the 1964 Declaration of Helsinki and its later amendments. Prior to data collection, all participants were informed of the purpose of this study and signed written consent forms were obtained to inform them of the rights, risks and advantages of participation. Participants gave informed consent to participate in the study before taking part.
Provenance and peer review Not commissioned; externally peer reviewed.

Data availability statement Data are available upon reasonable request. Data are available on the Dryad repository and the DOI number is https://doi.org/10.5061/ dryad.qfttdzojg.

Supplemental material This content has been supplied by the author(s). It has not been vetted by BMJ Publishing Group Limited (BMJ) and may not have been peer-reviewed. Any opinions or recommendations discussed are solely those of the author(s) and are not endorsed by BMJ. BMJ disclaims all liability and responsibility arising from any reliance placed on the content. Where the content includes any translated material, BMJ does not warrant the accuracy and reliability of the translations (including but not limited to local regulations, clinical guidelines, terminology, drug names and drug dosages), and is not responsible for any error and/or omissions arising from translation and adaptation or otherwise.

Open access This is an open access article distributed in accordance with the Creative Commons Attribution Non Commercial (CC BY-NC 4.0) license, which permits others to distribute, remix, adapt, build upon this work non-commercially, and license their derivative works on different terms, provided the original work is properly cited, appropriate credit is given, any changes made indicated, and the use is non-commercial. See: http://creativecommons.org/licenses/by-nc/4.0/.

ORCID iD

Yuxia Zhang http://orcid.org/0000-0003-4419-7004

\section{REFERENCES}

1 Donabedian A. The quality of care. How can it be assessed? JAMA 1988;260:1743-8.

2 Zamora D. Using patient satisfaction as a basis for reimbursement: political, financial, and philosophical implications. Creat Nurs 2012;18:118-23.

3 Hospital value-based purchasing. Centers for Medicare \& Medicaid Services, 2015. Available: https://www.cms.gov/Outreach-andEducation/Medicare-Learning-Network-MLN/MLNProducts/ 
Downloads/Hospital_VBPurchasing_Fact_Sheet_ICN907664.pdf [Accessed 22 Dec 2020].

4 Schneider J, Kaplan SH, Greenfield S, et al. Better physician-patient relationships are associated with higher reported adherence to antiretroviral therapy in patients with HIV infection. J Gen Intern Med 2004;19:1096-103.

5 Hill MH, Doddato T. Relationships among patient satisfaction, intent to return, and intent to recommend services provided by an academic nursing center. J Cult Divers 2002;9:108.

6 National Health and Family Planning Commission. Announcement of implementing the National healthcare improvement initiative, 2015. Available: http://www.nhc.gov.cn/yzygj/s3593g/201501/5584853c fa254d1aa4e38de0700891fa.shtml [Accessed 1 Oct 2020].

7 Batbaatar E, Dorjdagva J, Luvsannyam A, et al. Determinants of patient satisfaction: a systematic review. Perspect Public Health 2017;137:89-101.

8 Chang E, Hancock K, Chenoweth L, et al. The influence of demographic variables and ward type on elderly patients' perceptions of needs and satisfaction during acute hospitalization. Int J Nurs Pract 2003;9:191-201.

9 Mangelsdorff AD, Finstuen K. Patient satisfaction in military medicine: status and an empirical test of a model. Mil Med 2003;168:744-9.

10 Harris-Haywood S, Sylvia-Bobiak SN, Stange KC, et al. The association of how time is spent during outpatient visits and patient satisfaction: are there racial differences? J Natl Med Assoc 2007;99:1061-5.

11 Coroneos CJ, Lin Y-L, Sidey-Gibbons C, et al. Correlation between financial toxicity, quality of life, and patient satisfaction in an insured population of breast cancer surgical patients: a single-institution retrospective study. J Am Coll Surg 2021;232:253-63.

12 Min R, Li L, Zi C, et al. Evaluation of patient experience in countylevel public hospitals in China: a multicentred, cross-sectional study. BMJ Open 2019;9:e34225.

13 Greenslade JH, Jimmieson NL. Organizational factors impacting on patient satisfaction: a cross sectional examination of service climate and linkages to nurses' effort and performance. Int J Nurs Stud 2011;48:1188-98.

14 Ahmed F, Burt J, Roland M. Measuring patient experience: concepts and methods. Patient 2014;7:235-41.

15 Karam C. The evolution of patient satisfaction to patient experience. Front Health Serv Manage 2017:33:30-4.

16 Bleich SN, Ozaltin E, Murray CKL. How does satisfaction with the health-care system relate to patient experience? Bull World Health Organ 2009;87:271-8.

17 Park C, Zakare-Fagbamila RT, Dickson W. The limited influence of neurosurgeons' behavior on inpatient satisfaction: a retrospective multihospital analysis. J Neurosurg 2020;31:1-7.

18 Bjertnaes OA, Sjetne IS, Iversen HH. Overall patient satisfaction with hospitals: effects of patient-reported experiences and fulfilment of expectations. BMJ Qual Saf 2012;21:39-46.

19 The Lancet. 2020: unleashing the full potential of nursing. Lancet 20191879;394:1879.
20 Nursing and midwifery. World Health organization, 2020. Available: https://www.who.int/news-room/fact-sheets/detail/nursing-andmidwifery [Accessed 22 Dec 2020].

21 The National Health Commission of the People's Republic of China. China Health Statistics Yearbook[M]. Beijing: China Union Medical College Press, 2021.

22 Kutney-Lee A, McHugh MD, Sloane DM, et al. Nursing: a key to patient satisfaction. Health Aff 2009;28:w669-77.

23 Desborough J, Phillips C, Mills J, et al. Developing a positive patient experience with nurses in general practice: an integrated model of patient satisfaction and enablement. J Adv Nurs 2018;74:564-78.

24 Schmidt LA. Patients' perceptions of nurse staffing, nursing care, adverse events, and overall satisfaction with the hospital experience. Nurs Econ 2004;22:295.

25 von Elm E, Altman DG, Egger M, et al. The strengthening the reporting of observational studies in epidemiology (STROBE) statement: guidelines for reporting observational studies. Int J Surg 2014;12:1495-9.

26 Chen B. Sample Size Methodology for Multivariate Analysis Synthetic Estimate Method for Sample Size in Multivariate Analysis[J]. Injury Medicine 2012;1:58-60.

27 Desborough J, Bagheri N, Banfield M, et al. The impact of general practice nursing care on patient satisfaction and enablement in Australia: a mixed methods study. Int J Nurs Stud 2016;64:108-19.

28 Zhang Y, Li Q, Liu H. From patient satisfaction to patient experience: a call to action for nursing in China. J Nurs Manag 2020;28:450-6.

29 The Central People's Government of the People's Republic of China. The Ministry of health printed and distributed the working standards for implementing quality nursing care service in hospitals (trial), 2010. Available: http:// www.gov.cn/gzdt/2010-12/23/content_1771819.htm [Accessed 1 Oct 2020].

30 Senarat U, Gunawardena NS. Development of an instrument to measure patient perception of the quality of nursing care and related hospital services at the National Hospital of Sri Lanka. Asian Nurs Res 2011;5:71-80.

31 Cheraghi MA, Esmaeili M, Salsali M. Seeking humanizing care in patient-centered care process: a Grounded theory study. Holist Nurs Pract 2017;31:359-68.

32 Davey A, Carter M, Campbell JL. Priorities for young adults when accessing UK primary care: literature review. Prim Health Care Res Dev 2013;14:341-9.

33 Del Canale S, Louis DZ, Maio V, et al. The relationship between physician empathy and disease complications: an empirical study of primary care physicians and their diabetic patients in Parma, Italy. Acad Med 2012;87:1243-9.

34 Derksen F, Bensing J, Lagro-Janssen A. Effectiveness of empathy in general practice: a systematic review. Br J Gen Pract 2013;63:e76-84.

35 Sheard L, Marsh C, Mills T. Using patient experience data to develop a patient experience toolkit to improve hospital care: a mixed-methods study. Southampton (UK): NIHR Journals Library, 2019. 